\title{
ADAPTIVE LOW-GAIN INTEGRAL CONTROL OF MULTIVARIABLE WELL-POSED LINEAR SYSTEMS*
}

\author{
HARTMUT LOGEMANN ${ }^{\dagger}$ AND STUART TOWNLEY
}

\author{
To Ruth F. Curtain, on the occasion of her 60 th birthday
}

\begin{abstract}
The principle of low-gain integral control for finite-dimensional systems is well known. More recently, low-gain integral control results have been obtained for classes of infinite-dimensional systems. In this paper we show that integral control with a simple and natural adaptation of the integrator gain achieves tracking of constant reference signals for every exponentially stable, multivariable, well-posed, infinite-dimensional, linear system whose steady-state gain matrix has its spectrum in the open right-half plane. Our results considerably extend, improve, and simplify previous work by the authors [SIAM J. Control Optim. 35 (1997), pp. 78-116].
\end{abstract}

Key words. adaptive control, adaptive tracking, integral control, well-posed infinite-dimensional systems

AMS subject classifications. 93C20, 93C25, 93C40, 93D15, 93D21

PII. S0363012901396680

1. Introduction. There has been much interest over the last twenty-five years in low-gain integral control. Indeed, the following principle has become well established (see Davison [2], Lunze [7], and Morari [10]): closing the loop around an asymptotically stable, finite-dimensional, continuous-time plant, with transfer-function matrix $\mathbf{G}(s)$, compensated by an integrator $(k / s) I$ (see Figure 1), will result in an asymptotically stable closed-loop system which achieves asymptotic tracking of arbitrary constant reference signals, provided that the gain parameter $k>0$ is sufficiently small and the eigenvalues of the steady-state gain matrix $\mathbf{G}(0)$ have positive real parts, i.e.,

$$
\operatorname{spectrum}(\mathbf{G}(0)) \subset\{s \in \mathbb{C} \mid \operatorname{Re} s>0\} .
$$

This principle has been extended to various classes of infinite-dimensional systems; see Logemann and Townley [6] and the references therein. The generalization in [6] applies to so-called regular well-posed systems. We remark that the class of well-posed linear systems is the largest class of infinite-dimensional systems for which a well-developed state-space and frequency-domain theory exists; see Curtain and Weiss [3], Salamon [15], Staffans and Weiss [19], and Weiss [20], to mention just a few references. Wellposed systems are rather general in the sense that they capture most distributed parameter systems and all time-delay systems (retarded and neutral) which are of interest in applications. A well-posed system is called regular if the average of its step-response over $[0, t]$ converges as $t \rightarrow 0$ (equivalently, if its transfer functions $\mathbf{G}(s)$ converges as $s \rightarrow \infty$ on the positive real axis). Whilst the authors believe that any physically motivated well-posed linear system is regular, for a given well-posed system, regularity can be difficult to check.

* Received by the editors October 18, 2001; accepted for publication (in revised form) July 8, 2002; published electronically February 4, 2003. This work was supported in part by UK EPSRC grant GR/L78086.

http://www.siam.org/journals/sicon/41-6/39668.html

${ }^{\dagger}$ Department of Mathematical Sciences, University of Bath, Bath BA2 7AY, United Kingdom (hl@maths.bath.ac.uk).

${ }^{\ddagger}$ School of Mathematical Sciences, University of Exeter, Exeter EX4 4QE, United Kingdom (townley@maths.ex.ac.uk). 


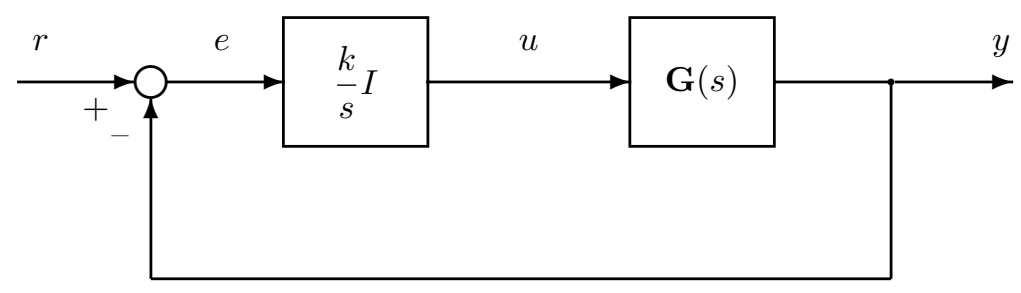

FIG. 1. Low-gain control system.

One of the main issues in the design of low-gain integral controllers is the tuning of the integrator gain $k$. There have been two basic approaches to the tuning problemeither steady-state data from the plant is used off-line to determine suitable ranges for the gain $k$ (see, for example, [2], Logemann, Ryan, and Townley [5], or [7]) or else simple on-line adaptive tuning of $k$ is used (see, for example, Miller and Davison [8,9] in the finite-dimensional case and [6] in the infinite-dimensional case). Of particular relevance here is a result in $[6]$ which shows that the adaptive integral controller

$$
\dot{u}(t)=\gamma^{-p}(t)(r-y(t)), \quad \dot{\gamma}(t)=\|r-y(t)\|^{2}
$$

achieves asymptotic tracking of arbitrary constant reference signals $r$, provided that the following three assumptions are satisfied:

(i) the plant is an exponentially stable, regular, well-posed, infinite-dimensional system;

(ii) the steady-state gain matrix $\mathbf{G}(0)$ is symmetric and positive definite;

(iii) the parameter $p$ in $(1.2)$ satisfies $p \in(0,1 / 2)$.

We note that earlier work by Cook [1] shows that in the finite-dimensional singleinput single-output case, assumption (iii) can be relaxed to $p \in(0,1]$. Of course, the symmetry assumption in (ii) is restrictive and highly nonrobust, essentially limiting the applications of the above result to single-input single-output systems. The main result of this note (Theorem 3.1) shows that assumption (ii) can be replaced by the considerably weaker assumption (1.1), that the regularity assumption in (i) can be dropped, and that (iii) can replaced by $p \in(0,1]$. Furthermore, in comparing the results we present here to those in [6], the proofs are dramatically simplified and more importantly give a clearer insight into the structure of the resulting closed-loop system. We emphasize that our main result is new even in the finite-dimensional case.

Notation. $\mathbb{R}_{+}:=[0, \infty)$; for $\alpha \in \mathbb{R}$, set $\mathbb{C}_{\alpha}:=\{s \in \mathbb{C} \mid \operatorname{Re} s>\alpha\}$; let $Z$ be a real or complex Banach space; for $\alpha \in \mathbb{R}$, we define the exponentially weighted $L^{p}$-space $L_{\alpha}^{p}\left(\mathbb{R}_{+}, Z\right):=\left\{f \in L_{\text {loc }}^{p}\left(\mathbb{R}_{+}, Z\right) \mid f(\cdot) \exp (-\alpha \cdot) \in L^{p}\left(\mathbb{R}_{+}, Z\right)\right\}$ and endow it with the norm $\|f\|_{p, \alpha}:=\left\|e^{-\alpha \cdot} f(\cdot)\right\|_{L^{p}}$; let $H^{2}\left(\mathbb{C}_{\alpha}, Z\right)$ denote the Hardy-Lebesgue space of square-integrable holomorphic functions defined on $\mathbb{C}_{\alpha}$ with values in $Z ; H^{\infty}\left(\mathbb{C}_{\alpha}, Z\right)$ denotes the space of bounded holomorphic functions defined on $\mathbb{C}_{\alpha}$ with values in $Z ; \mathcal{B}\left(Z_{1}, Z_{2}\right)$ denotes the space of bounded linear operators from a Banach space $Z_{1}$ to a Banach space $Z_{2}$; we write $\mathcal{B}(Z)$ for $\mathcal{B}(Z, Z)$; let $A: \operatorname{dom}(A) \subset Z \rightarrow Z$ be a linear operator, where $\operatorname{dom}(A)$ denotes the domain of $A$; the resolvent set of $A$ and the spectrum of $A$ is denoted by $\varrho(A)$ and $\sigma(A)$, respectively; the Laplace transform is denoted by $\mathfrak{L}$.

2. Preliminaries on well-posed sytems. There are a number of equivalent definitions of well-posed systems; see [3, 14, 15, 16, 17, 18, 19, 20, 21]. We will be 
brief in the following and refer the reader to the above references for more details. Throughout this section, we shall be considering a well-posed system $\Sigma$ with statespace $X$, input space $\mathbb{R}^{m}$, and output space $\mathbb{R}^{m}$, generating operators $(A, B, C)$, inputoutput operator $G$, and transfer function $\mathbf{G}$. Here $X$ is a real Hilbert space with norm denoted by $\|\cdot\|, A$ is the generator of a strongly continuous semigroup $\mathbf{T}=\left(\mathbf{T}_{t}\right)_{t \geq 0}$ on $X, B \in \mathcal{B}\left(\mathbb{R}^{m}, X_{-1}\right)$, and $C \in \mathcal{B}\left(X_{1}, \mathbb{R}^{m}\right)$, where $X_{1}$ denotes the space $\operatorname{dom}(A)$ endowed with the norm $\|x\|_{1}:=\|x\|+\|A x\|$ (the graph norm of $A$ ), whilst $X_{-1}$ denotes the completion of $X$ with respect to the norm $\|x\|_{-1}=\left\|(\lambda I-A)^{-1} x\right\|$, where $\lambda \in \varrho(A)$ (different choices of $\lambda$ lead to equivalent norms). Clearly, $X_{1} \subset X \subset X_{-1}$ and the canonical injections are bounded and dense. The semigroup $\mathbf{T}$ restricts to a strongly continuous semigroup on $X_{1}$ and extends to a strongly continuous semigroup on $X_{-1}$ with the exponential growth constant being the same on all three spaces; the generator of the restriction (extension) of $\mathbf{T}$ is a restriction (extension) of $A$; we shall use the same symbol $\mathbf{T}$ (respectively, $A$ ) for the original semigroup (respectively, generator) and the associated restrictions and extensions: with this convention, we may write $A \in \mathcal{B}\left(X, X_{-1}\right)$ (considered as a generator on $X_{-1}$, the domain of $A$ is $X$ ). Moreover, the operator $B$ is an admissible control operator for $\mathbf{T}$, i.e., for each $t \in \mathbb{R}_{+}$ there exists $\alpha_{t} \geq 0$ such that

$$
\left\|\int_{0}^{t} \mathbf{T}_{t-\tau} B u(\tau) d \tau\right\| \leq \alpha_{t}\|u\|_{L^{2}\left([0, t], \mathbb{R}^{m}\right)} \quad \forall u \in L^{2}\left([0, t], \mathbb{R}^{m}\right) .
$$

The operator $C$ is an admissible observation operator for $\mathbf{T}$, i.e., for each $t \in \mathbb{R}_{+}$there exists $\beta_{t} \geq 0$ such that

$$
\left(\int_{0}^{t}\left\|C \mathbf{T}_{\tau} x\right\|^{2} d \tau\right)^{1 / 2} \leq \beta_{t}\|x\| \quad \forall x \in X_{1} .
$$

The control operator $B$ is said to be bounded if it is so as a map from the input space $\mathbb{R}^{m}$ to the state-space $X$; otherwise, it is said to be unbounded; the observation operator $C$ is said to be bounded if it can be extended continuously to $X$; otherwise, $C$ is said to be unbounded.

The so-called $\Lambda$-extension $C_{\Lambda}$ of $C$ is defined by

$$
C_{\Lambda} x=\lim _{s \rightarrow \infty, s \in \mathbb{R}} C s(s I-A)^{-1} x,
$$

with $\operatorname{dom}\left(C_{\Lambda}\right)$ consisting of all $x \in X$ for which the above limit exists. For every $x \in X, \mathbf{T}_{t} x \in \operatorname{dom}\left(C_{\Lambda}\right)$ for a.a. $t \in \mathbb{R}_{+}$, and, if $\omega>\omega(\mathbf{T})$, then $C_{\Lambda} \mathbf{T} x \in L_{\omega}^{2}\left(\mathbb{R}_{+}, \mathbb{R}^{m}\right)$, where

$$
\omega(\mathbf{T}):=\lim _{t \rightarrow \infty} \frac{1}{t} \ln \left\|\mathbf{T}_{t}\right\|
$$

denotes the exponential growth constant of $\mathbf{T}$. The transfer function $\mathbf{G}$ satisfies

$$
\frac{1}{s-\lambda}(\mathbf{G}(s)-\mathbf{G}(\lambda))=-C(s I-A)^{-1}(\lambda I-A)^{-1} B \quad \forall s, \lambda \in \mathbb{C}_{\omega(\mathbf{T})}, s \neq \lambda,
$$

and $\mathbf{G} \in H^{\infty}\left(\mathbb{C}_{\omega}, \mathbb{R}^{m \times m}\right)$ for every $\omega>\omega(\mathbf{T})$. Moreover, the input-output operator $G: L_{\text {loc }}^{2}\left(\mathbb{R}_{+}, \mathbb{R}^{m}\right) \rightarrow L_{\text {loc }}^{2}\left(\mathbb{R}_{+}, \mathbb{R}^{m}\right)$ is continuous and shift-invariant; for every $\omega>$ $\omega(\mathbf{T}), G \in \mathcal{B}\left(L_{\omega}^{2}\left(\mathbb{R}_{+}, \mathbb{R}^{m}\right)\right)$ and

$$
(\mathfrak{L}(G u))(s)=\mathbf{G}(s)(\mathfrak{L}(u))(s) \quad \forall s \in \mathbb{C}_{\omega}, \quad \forall u \in L_{\omega}^{2}\left(\mathbb{R}_{+}, \mathbb{R}^{m}\right) .
$$


In the following, let $\lambda \in \mathbb{C}_{\omega(\mathbf{T})}$ be fixed but arbitrary. For $x^{0} \in X$ and $u \in$ $L_{\text {loc }}^{2}\left(\mathbb{R}_{+}, \mathbb{R}^{m}\right)$, let $x$ and $y$ denote the state and output functions of $\Sigma$, respectively, corresponding to the initial condition $x(0)=x^{0} \in X$ and the input function $u$. Then $x(t)=\mathbf{T}_{t} x^{0}+\int_{0}^{t} \mathbf{T}_{t-\tau} B u(\tau) d \tau$ for all $t \in \mathbb{R}_{+}, x(t)-(\lambda I-A)^{-1} B u(t) \in \operatorname{dom}\left(C_{\Lambda}\right)$ for a.a. $t \in \mathbb{R}_{+},{ }^{1}$ and

$$
\begin{aligned}
& \dot{x}(t)=A x(t)+B u(t), \quad x(0)=x^{0}, \quad \text { for a.a. } t \in \mathbb{R}_{+}, \\
& y(t)=C_{\Lambda}\left(x(t)-(\lambda I-A)^{-1} B u(t)\right)+\mathbf{G}(\lambda) u(t), \quad \text { for a.a. } t \geq 0 .
\end{aligned}
$$

Of course, the differential equation (2.2a) has to be interpreted in $X_{-1}$. Note that the output equation $(2.2 \mathrm{~b})$ yields the following formula for the input-output operator $G$ :

$$
\begin{aligned}
(G u)(t)=C_{\Lambda}\left[\int_{0}^{t} \mathbf{T}_{t-\tau} B u(\tau) d \tau\right. & \left.-(\lambda I-A)^{-1} B u(t)\right]+\mathbf{G}(\lambda) u(t) \\
\forall u & \in L_{\mathrm{loc}}^{2}\left(\mathbb{R}_{+}, \mathbb{R}^{m}\right), \text { for a.a. } t \in \mathbb{R}_{+} .
\end{aligned}
$$

In the following, we identify $\Sigma$ and (2.2) and refer to (2.2) as a well-posed system. We say that the well-posed system $(2.2)$ is exponentially stable if $\omega(\mathbf{T})<0$. If the well-posed system (2.2) is regular, i.e., the following limit

$$
\lim _{s \rightarrow \infty, s \in \mathbb{R}} \mathbf{G}(s)=D
$$

exists, then $x(t) \in \operatorname{dom}\left(C_{\Lambda}\right)$ for a.a. $t \in \mathbb{R}_{+}$, the output equation (2.2b) simplifies to

$$
y(t)=C_{\Lambda} x(t)+D u(t), \quad \text { for a.a. } t \geq 0,
$$

and

$$
(G u)(t)=C_{\Lambda} \int_{0}^{t} \mathbf{T}_{t-\tau} B u(\tau) d \tau+D u(t) \quad \forall u \in L_{\mathrm{loc}}^{2}\left(\mathbb{R}_{+}, \mathbb{R}^{m}\right), \text { for a.a. } t \in \mathbb{R}_{+} .
$$

Moreover, in the regular case, we have that $(s I-A)^{-1} B \mathbb{R}^{m} \subset \operatorname{dom}\left(C_{\Lambda}\right)$ for all $s \in \varrho(A)$ and

$$
\mathbf{G}(s)=C_{\Lambda}(s I-A)^{-1} B+D \quad \forall s \in \mathbb{C}_{\omega(\mathbf{T})} .
$$

The matrix $D \in \mathbb{R}^{m \times m}$ is called the feedthrough matrix of (2.2). We mention that if the control operator $B$ or the observation operator $C$ is bounded, then (2.2) is regular.

3. Main result. We consider adaptive low-gain integral control of an exponentially stable well-posed system of the form (2.2). By exponential stability, we may assume w.l.o.g. that $\lambda=0$ in $(2.2 \mathrm{~b})$, and hence the plant equations are given by

$$
\begin{aligned}
& \dot{x}=A x+B u, \quad x(0)=x^{0} \in X, \\
& y=C_{\Lambda}\left(x+A^{-1} B u\right)+\mathbf{G}(0) u .
\end{aligned}
$$

Let $r \in \mathbb{R}^{m}$ be a given reference vector and consider the following simple adaptive low-gain integral controller:

$$
\begin{aligned}
& \dot{u}=\gamma^{-p}(r-y), \quad u(0)=u^{0} \in \mathbb{R}^{m}, \\
& \dot{\gamma}=\|r-y\|^{2}, \quad \gamma(0)=\gamma^{0}>0,
\end{aligned}
$$

\footnotetext{
${ }^{1}$ It was stated in [21] (without proof) that, for arbitrary $u \in L_{\text {loc }}^{2}\left(\mathbb{R}_{+}, \mathbb{R}^{m}\right), x(t)-(\lambda I-$ $A)^{-1} B u(t) \in \operatorname{dom}\left(C_{\Lambda}\right)$ for a.a. $t \in \mathbb{R}_{+}$and the output formula (2.2b) holds. The proof can be found in [19].
} 
where $0<p \leq 1$. The closed-loop system is then given by

$$
\begin{aligned}
& \dot{x}=A x+B u, \quad x(0)=x^{0} \in X, \\
& \dot{u}=\gamma^{-p}\left(r-C_{\Lambda}\left(x+A^{-1} B u\right)-\mathbf{G}(0) u\right), \quad u(0)=u^{0} \in \mathbb{R}^{m}, \\
& \dot{\gamma}=\left\|r-C_{\Lambda}\left(x+A^{-1} B u\right)-\mathbf{G}(0) u\right\|^{2}, \quad \gamma(0)=\gamma^{0}>0 .
\end{aligned}
$$

Let $T>0$. A continuous function $(x, u, \gamma):[0, T) \rightarrow X \times \mathbb{R}^{m} \times \mathbb{R}$ is called a solution of $(3.3)$ if $(x(0), u(0), \gamma(0))=\left(x^{0}, u^{0}, \gamma^{0}\right),(x, u, \gamma)$ is absolutely continuous on $[0, t]$ as a $\left(X_{-1} \times \mathbb{R}^{m} \times \mathbb{R}\right)$-valued function for every $t \in(0, T)$ and the differential equations in (3.3) are satisfied almost everywhere on $[0, T)$.

Our main result, Theorem 3.1, shows that the controller (3.2) achieves tracking of constant reference signals for all exponentially stable well-posed systems (3.1) whose steady-state gain matrix $\mathbf{G}(0)$ satisfies $\sigma(\mathbf{G}(0)) \subset \mathbb{C}_{0}$.

THEOREM 3.1. Assume that the well-posed system (3.1) is exponentially stable with $\sigma(\mathbf{G}(0)) \subset \mathbb{C}_{0}$ and that $0<p \leq 1$ in (3.2). Let $r \in \mathbb{R}^{m}$ be given and define $u^{r}:=[\mathbf{G}(0)]^{-1} r$. Then, for all $\left(x^{0}, u^{0}, \gamma^{0}\right) \in X \times \mathbb{R}^{m} \times(0, \infty)$, there exists a unique solution $(x, u, \gamma): \mathbb{R}_{+} \rightarrow X \times \mathbb{R}^{m} \times(0, \infty)$ of the closed-loop system (3.3) and the following statements hold:

(1) $\lim _{t \rightarrow \infty} \gamma(t)=\gamma^{\infty}<\infty$;

(2) $u-u^{r} \in L^{2}\left(\mathbb{R}_{+}, \mathbb{R}^{m}\right)$ and $\lim _{t \rightarrow \infty} u(t)=u^{r}$;

(3) $x+A^{-1} B u^{r} \in L^{2}\left(\mathbb{R}_{+}, X\right)$ and $\lim _{t \rightarrow \infty}\left\|x(t)+A^{-1} B u^{r}\right\|=0$;

(4) $e:=r-y \in L^{2}\left(\mathbb{R}_{+}, \mathbb{R}^{m}\right)$ and $e$ admits a decomposition of the form $e=$ $e_{1}+e_{2}$, where $e_{1} \in C\left(\mathbb{R}_{+}, \mathbb{R}^{m}\right)$, $e_{2} \in L_{\omega}^{2}\left(\mathbb{R}_{+}, \mathbb{R}^{m}\right)$ for every $\omega>\omega(\mathbf{T})$, and $\lim _{t \rightarrow \infty} e_{1}(t)=0$; moreover, if there exists $t_{0} \geq 0$ such that $\mathbf{T}_{t_{0}}\left(A x^{0}+B u^{0}\right) \in$ $X$, then $e \in C\left(\left[t_{0}, \infty\right), \mathbb{R}^{m}\right)$ and $\lim _{t \rightarrow \infty} e(t)=0$.

Proof. As in [6] it can be proved that there exists a unique maximally defined solution $(x, u, \gamma):[0, T) \rightarrow X \times \mathbb{R}^{m} \times(0, \infty)$ to the closed-loop system (3.3), where $T=\infty$ if $\gamma$ is bounded on $[0, T)$. To analyze the stability of the closed-loop system (3.3), we use a change of coordinates. Define

$$
z(t):=x(t)+A^{-1} B u(t), \quad v(t):=u(t)-u^{r} ;
$$

it follows from (3.1) and (3.2) that

$$
\begin{aligned}
& \dot{z}(t)=A z(t)+\gamma^{-p}(t) A^{-1} B e(t), \quad \text { for a.a. } t \in[0, T), \\
& \dot{v}(t)=\gamma^{-p}(t) e(t), \quad \text { for a.a. } t \in[0, T),
\end{aligned}
$$

where

$$
e(t):=r-y(t)=-\left(C_{\Lambda} z(t)+\mathbf{G}(0) v(t)\right), \quad \text { for a.a. } t \in[0, T) .
$$

Of course, the derivative on the left-hand side of (3.5a) has to be interpreted in $X_{-1}$. There are two advantages to viewing the closed-loop system in the coordinates (3.4): the unbounded $B$ in (3.1a) is replaced by a bounded $A^{-1} B$ and it turns out that the stability of $-\mathbf{G}(0)$ is easier to exploit in the $(z, v)$-coordinates than in the $(x, u)$ coordinates.

To proceed, we use the stability of $-\mathbf{G}(0)$ to obtain the existence of $Q=Q^{T} \in$ $\mathbb{R}^{m \times m}$ with $Q>0$ such that

$$
Q \mathbf{G}(0)+\mathbf{G}(0)^{T} Q=I .
$$


Furthermore, using the exponential stability of $\mathbf{T}$ and the admissibility of $C$, a standard argument (see the appendix) shows that there exists $P=P^{*} \in \mathcal{B}(X)$ with $P \geq 0$ and such that

$$
\left\langle A x_{1}, P x_{2}\right\rangle+\left\langle P x_{1}, A x_{2}\right\rangle=-\left\langle x_{1}, x_{2}\right\rangle-\left\langle C x_{1}, C x_{2}\right\rangle \quad \forall x_{1}, x_{2} \in X_{1} .
$$

Our aim is to show that $\gamma$ is bounded on $[0, T)$, from which we will deduce that $T=\infty$ and that statements (1)-(4) hold. To this end, define a function $V:[0, T) \rightarrow \mathbb{R}_{+}$by

$$
V(t)=\langle z(t), P z(t)\rangle+\langle v(t), Q v(t)\rangle \quad \forall t \in[0, T),
$$

where the first inner product is taken in $X$ and the second inner product is the standard inner product in $\mathbb{R}^{m}$. Since, due to lack of regularity of $z$ as a $X$-valued function, $V$ is in general not differentiable, we adopt an approximation argument. Define

$$
z_{n}(t):=\mathbf{T}_{t} z_{n}^{0}+A^{-1} \int_{0}^{t} \mathbf{T}_{t-\tau} B \gamma^{-p}(\tau) e(\tau) d \tau \quad \forall t \in[0, T)
$$

where $z_{n}^{0} \in X_{1}$ is such that $z_{n}^{0} \rightarrow z(0)=x^{0}+A^{-1} B u^{0}$ as $n \rightarrow \infty$. Clearly, $z_{n}(t) \in X_{1}$ for all $t \in[0, T)$. It follows from the admissibility of $B$ and well-known results on abstract Cauchy problems (see [11, p. 109]) that $z_{n}$ is absolutely continuous as a $X$-valued function and

$$
\dot{z}_{n}(t)=A z_{n}(t)+\gamma^{-p}(t) A^{-1} B e(t), \quad \text { for a.a. } t \in[0, T) .
$$

Therefore, the function

$$
V_{n}:[0, T) \rightarrow \mathbb{R}_{+}, \quad t \mapsto\left\langle z_{n}(t), P z_{n}(t)\right\rangle+\langle v(t), Q v(t)\rangle
$$

is absolutely continuous. Invoking (3.5)-(3.8), we compute the derivative of $V_{n}$ to be

$$
\dot{V}_{n}=-\left\|z_{n}\right\|^{2}-\left\|C z_{n}\right\|^{2}+2 \gamma^{-p}\left\langle z_{n}, P A^{-1} B e\right\rangle-\gamma^{-p}\|v\|^{2}-2 \gamma^{-p}\left\langle v, Q C_{\Lambda} z\right\rangle .
$$

Integrating (3.9) from $s$ to $t$, where $0 \leq s \leq t<T$, we obtain

$$
\begin{aligned}
V_{n}(t)- & V_{n}(s)=-\int_{s}^{t}\left(\left\|z_{n}(\tau)\right\|^{2}+\left\|C z_{n}(\tau)\right\|^{2}+\gamma^{-p}\|v(\tau)\|^{2}\right. \\
& \left.-2 \gamma^{-p}(\tau)\left\langle z_{n}(\tau), P A^{-1} B e(\tau)\right\rangle+2 \gamma^{-p}(\tau)\left\langle v(\tau), Q C_{\Lambda} z(\tau)\right\rangle\right) d \tau
\end{aligned}
$$

It follows from (3.5a) that $z(t):=\mathbf{T}_{t} z(0)+A^{-1} \int_{0}^{t} \mathbf{T}_{t-\tau} B \gamma^{-p}(\tau) e(\tau) d \tau$, showing that $z(t)-z_{n}(t)=\mathbf{T}_{t}\left(z(0)-z_{n}^{0}\right)$ for all $t \in[0, T)$ and hence for all $t \in[0, T)$

$\lim _{n \rightarrow \infty}\left\|z(t)-z_{n}(t)\right\|=0, \quad \lim _{n \rightarrow \infty}\left\|z-z_{n}\right\|_{L^{2}(0, t)}=0, \quad \lim _{n \rightarrow \infty}\left\|C_{\Lambda} z-C_{\Lambda} z_{n}\right\|_{L^{2}(0, t)}=0$,

where the last limit follows from the admissibility of $C$. Consequently, letting $n \rightarrow \infty$ in (3.10), we may conclude that

$$
\begin{aligned}
V(t)- & V(s)=-\int_{s}^{t}\left(\|z(\tau)\|^{2}+\left\|C_{\Lambda} z(\tau)\right\|^{2}+\gamma^{-p}\|v(\tau)\|^{2}\right. \\
& \left.-2 \gamma^{-p}(\tau)\left\langle z(\tau), P A^{-1} B e(\tau)\right\rangle+2 \gamma^{-p}(\tau)\left\langle v(\tau), Q C_{\Lambda} z(\tau)\right\rangle\right) d \tau .
\end{aligned}
$$


Denoting the integrand on the right-hand side of (3.11) by $f(\tau)$ and using (3.6), routine estimates give

$$
\begin{aligned}
& f \geq\|z\|^{2}+\left\|C_{\Lambda} z\right\|^{2}+c_{1} \gamma^{-p}\|\mathbf{G}(0) v\|^{2} \\
& \quad-c_{2} \gamma^{-p}\left(\|z\|\left\|C_{\Lambda} z\right\|+\|z\|\|\mathbf{G}(0) v\|+\left\|C_{\Lambda} z\right\|\|\mathbf{G}(0) v\|\right) \\
& \geq\|z\|^{2}+\left\|C_{\Lambda} z\right\|^{2}+c_{1} \gamma^{-p}\|\mathbf{G}(0) v\|^{2}-c_{2} \gamma^{-p}\left(\|z\|^{2}+\left\|C_{\Lambda} z\right\|^{2}\right) \\
& \quad-c_{2} \gamma^{-p}\left(\lambda\|z\|^{2}+\|\mathbf{G}(0) v\|^{2} / \lambda\right)-c_{2} \gamma^{-p}\left(\lambda\left\|C_{\Lambda} z\right\|^{2}+\|\mathbf{G}(0) v\|^{2} / \lambda\right),
\end{aligned}
$$

where $c_{1}, c_{2}>0$ are suitable constants and $\lambda>0$ is arbitrary. In the following we choose

$$
\lambda=4 c_{2} / c_{1} .
$$

We show that $\gamma$ is bounded on $[0, T)$. If

$$
\gamma^{-p}(\tau)>1 /\left[2 c_{2}(1+\lambda)\right] \quad \forall t \in[0, T),
$$

then there is nothing to prove. So assume that (3.14) does not hold. Then, by monotonicity of $\gamma$, there exists $t_{0} \in[0, T)$ such that

$$
\gamma^{-p}(\tau) \leq 1 /\left[2 c_{2}(1+\lambda)\right] \quad \forall t \in\left[t_{0}, T\right) .
$$

Combining this with (3.12) and (3.13) yields

$$
\begin{array}{rlrl}
f(\tau) & \geq\left(\|z(\tau)\|^{2}+\left\|C_{\Lambda} z(\tau)\right\|^{2}+c_{1} \gamma^{-p}(\tau)\|\mathbf{G}(0) v(\tau)\|^{2}\right) / 2 & \\
& \geq c_{3} \gamma^{-p}(\tau)\left(\left\|C_{\Lambda} z(\tau)\right\|^{2}+\|\mathbf{G}(0) v(\tau)\|^{2}\right) \quad \forall t \in\left[t_{0}, T\right),
\end{array}
$$

where $c_{3}>0$ is a suitable constant. Noting that

$$
\|r-y\|^{2}=\|e\|^{2}=\left\|C_{\Lambda} z+\mathbf{G}(0) v\right\|^{2} \leq 2\left(\left\|C_{\Lambda} z\right\|^{2}+\|\mathbf{G}(0) v\|^{2}\right),
$$

we see that there exists a constant $c_{4}>0$ such that

$$
f(\tau) \geq c_{4} \gamma^{-p}(\tau)\|r-y(\tau)\|^{2} \quad \forall \tau \in\left[t_{0}, T\right) .
$$

Therefore, by (3.11),

$$
V(t)-V\left(t_{0}\right)=-\int_{t_{0}}^{t} f(\tau) d \tau \leq-c_{4} \int_{t_{0}}^{t} \gamma^{-p}(\tau)\|r-y(\tau)\|^{2} d \tau \quad \forall t \in\left[t_{0}, T\right)
$$

But $\dot{\gamma}=\|r-y\|^{2}$, and hence, by (3.15),

$$
\int_{\gamma\left(t_{0}\right)}^{\gamma(t)} w^{-p} d w=\int_{t_{0}}^{t} \gamma^{-p}(\tau) \dot{\gamma}(\tau) d \tau \leq V\left(t_{0}\right) / c_{4} \quad \forall t \in\left[t_{0}, T\right) .
$$

Since $0<p \leq 1$, this inequality implies that $\gamma$ is bounded on $[0, T)$, showing that $T=\infty$ and also establishing statement (1).

To prove statements (2) and (3) note that

$$
e \in L^{2}\left(\mathbb{R}_{+}, \mathbb{R}^{m}\right),
$$


which follows immediately from the boundedness of $\gamma$ and the fact that $\dot{\gamma}=\|e\|^{2}$. Since $\left(A, A^{-1} B, C\right)$ are the generators of an exponentially stable regular system (with zero feedthrough), it follows from (3.5a) and (3.16) that

$$
\lim _{t \rightarrow \infty}\|z(t)\|=0, \quad z \in L^{2}\left(\mathbb{R}_{+}, X\right), \quad C_{\Lambda} z \in L^{2}\left(\mathbb{R}_{+}, \mathbb{R}^{m}\right) .
$$

Using that $C_{\Lambda} z \in L^{2}\left(\mathbb{R}_{+}, \mathbb{R}^{m}\right)$, it follows from (3.6), (3.16), and the invertibility of $\mathbf{G}(0)$ that $v \in L^{2}\left(\mathbb{R}_{+}, \mathbb{R}^{m}\right)$. But by $(3.5 \mathrm{~b})$ and (3.16) we also have that $\dot{v} \in$ $L^{2}\left(\mathbb{R}_{+}, \mathbb{R}^{m}\right)$, showing that $\lim _{t \rightarrow \infty} v(t)=0$ and completing the proof of statement (2). Since $x+A^{-1} B u^{r}=z+A^{-1} B\left(u^{r}-u\right)$, statement (3) follows from statement (2) and (3.17).

To prove statement (4), we first note that we have already shown that $e \in$ $L^{2}\left(\mathbb{R}_{+}, \mathbb{R}^{m}\right)$, and so

$$
\dot{u} \in L^{2}\left(\mathbb{R}_{+}, \mathbb{R}^{m}\right) .
$$

We recall that $G$ denotes the input-output operator of (3.1). Define a shift-invariant operator $H: L_{\text {loc }}^{2}\left(\mathbb{R}_{+}, \mathbb{R}^{m}\right) \rightarrow L_{\text {loc }}^{2}\left(\mathbb{R}_{+}, \mathbb{R}^{m}\right)$ by setting

$$
(H w)(t):=\int_{0}^{t}((G w)(\tau)-\mathbf{G}(0) w(\tau)) d \tau \quad \forall w \in L_{\mathrm{loc}}^{2}\left(\mathbb{R}_{+}, \mathbb{R}^{m}\right) \quad \forall t \in \mathbb{R}_{+} .
$$

The transfer function $\mathbf{H}$ of $H$ is given by $\mathbf{H}(s)=(\mathbf{G}(s)-\mathbf{G}(0)) / s$. Clearly, for every $\omega>\omega(\mathbf{T})$

$$
\mathbf{H} \in H^{2}\left(\mathbb{C}_{\omega}, \mathbb{C}^{m \times m}\right) \cap H^{\infty}\left(\mathbb{C}_{\omega}, \mathbb{C}^{m \times m}\right),
$$

showing in particular that $H$ is bounded, i.e., $H \in \mathcal{B}\left(L^{2}\left(\mathbb{R}_{+}, \mathbb{R}^{m}\right)\right)$. Using that $G$ commutes with the integration operator (by shift-invariance), a routine calculation gives

$$
G u=H \dot{u}+\mathbf{G}(0) u+G\left(u^{0} \theta\right)-\mathbf{G}(0) u^{0},
$$

where $\theta$ denotes the unit-step function. Invoking the output formula $y=C_{\Lambda} \mathbf{T} x^{0}+G u$, we may write $e=r-y=e_{1}+e_{2}$, where

$$
e_{1}:=\mathbf{G}(0)\left(u^{r}-u\right)-H \dot{u}, \quad e_{2}:=\mathbf{G}(0) u^{0}-G\left(u^{0} \theta\right)-C_{\Lambda} \mathbf{T} x^{0} .
$$

Clearly, $e_{1}$ is continuous. Using (3.18) and the boundedness of $H$ and $G$, it follows that $H \dot{u}$ and $(d / d t)(H \dot{u})$ are in $L^{2}\left(\mathbb{R}_{+}, \mathbb{R}^{m}\right)$ and hence $\lim _{t \rightarrow \infty}(H \dot{u})(t)=0$. Combining this with statement (2) shows that $\lim _{t \rightarrow \infty} e_{1}(t)=0$. Let $\omega>\omega(\mathbf{T})$. To prove that $e_{2} \in L_{\omega}^{2}\left(\mathbb{R}_{+}, \mathbb{R}^{m}\right)$, it is sufficient to show that $g:=\mathbf{G}(0) u^{0}-G\left(u^{0} \theta\right) \in L_{\omega}^{2}\left(\mathbb{R}_{+}, \mathbb{R}^{m}\right)$. But $(\mathfrak{L} g)(s)=-\mathbf{H}(s) u^{0}$, and so $\mathfrak{L} g \in H^{2}\left(\mathbb{C}_{\omega}, \mathbb{C}^{m}\right)$, which in turn implies (by a wellknown result of Paley and Wiener, see $\left[12\right.$, p. 405]) that $g \in L_{\omega}^{2}\left(\mathbb{R}_{+}, \mathbb{R}^{m}\right)$. Finally, assume that there exists $t_{0} \geq 0$ such that $\mathbf{T}_{t_{0}}\left(A x^{0}+B u^{0}\right) \in X$. Taking the Laplace transform of $e_{2}$ gives

$$
\left(\mathfrak{L} e_{2}\right)(s)=(\mathbf{G}(0)-\mathbf{G}(s)) u^{0} / s-C(s I-A)^{-1} x^{0} \quad \forall s \in \mathbb{C}_{\omega} .
$$

Invoking (2.1) leads to

$$
\begin{aligned}
\left(\mathfrak{L} e_{2}\right)(s) & =-C(s I-A)^{-1} A^{-1} B u^{0}-C(s I-A)^{-1} x^{0} \\
& =-C(s I-A)^{-1} A^{-1}\left(A x^{0}+B u^{0}\right) \quad \forall s \in \mathbb{C}_{\omega},
\end{aligned}
$$


implying that $e_{2}(t)=-C_{\Lambda} \mathbf{T}_{t} A^{-1}\left(A x^{0}+B u^{0}\right)$ for a.a. $t \in \mathbb{R}_{+}$. Hence, since $\mathbf{T}_{t_{0}}\left(A x^{0}+\right.$ $\left.B u^{0}\right) \in X$,

$$
e_{2}(t)=-C \mathbf{T}_{t-t_{0}} A^{-1} \mathbf{T}_{t_{0}}\left(A x^{0}+B u^{0}\right), \quad \text { for a.a. } t \geq t_{0},
$$

showing that $e_{2}$, and hence $e$, is continuous on $\left[t_{0}, \infty\right)$ and $\lim _{t \rightarrow \infty} e_{2}(t)=0=$ $\lim _{t \rightarrow \infty} e(t)$.

Remark 3.2. (1) Statement (4) in Theorem 3.1 shows that the tracking error $e$ becomes small in the sense that $e=e_{1}+e_{2}$, where $e_{1}(t)$ converges to 0 as $t \rightarrow \infty$, $e_{1} \in L^{2}\left(\mathbb{R}_{+}, \mathbb{R}^{m}\right)$, and $e_{2} \in L_{\omega}^{2}\left(\mathbb{R}_{+}, \mathbb{R}^{m}\right)$ for $\omega>\omega(\mathbf{T})$. This implies, in particular, "tracking in measure," i.e., for all $\varepsilon>0$ we have that

$$
\lim _{\tau \rightarrow \infty} \mu_{L}(\{t \geq \tau \mid\|e(t)\| \geq \varepsilon\})=0,
$$

where $\mu_{L}$ denotes the Lebesgue measure on $\mathbb{R}_{+}$. The last part of statement (4) shows that "asymptotic tracking" (i.e., $\lim _{t \rightarrow \infty} e(t)=0$ ) is guaranteed, provided that $\mathbf{T}_{t_{0}}\left(A x^{0}+B u^{0}\right) \in X$ for some $t_{0} \geq 0$ (which, for example, is the case if $\mathbf{T}$ is holomorphic).

(2) Under the conditions of Theorem 3.1, it is easy to see that if $\mathbf{T}_{t_{0}} x^{0} \in X_{1}$ for some $t_{0} \geq 0$ and the convolution kernel of $G$ is a finite (matrix-valued) Borel measure, then $\lim _{t \rightarrow \infty} e(t)=0$.

(3) Combining the above change of coordinates technique with the approach in [4], it can be shown that the adaptation law (3.2b) can be generalized to $\dot{\gamma}(t)=\|r-y(t)\|^{q}$ for arbitrary $q \geq 1$.

(4) Theorem 3.1 remains true if we replace the finite-dimensional input space $\mathbb{R}^{m}$ by an arbitrary real Hilbert space; the proof carries over word for word to this more general situation.

(5) Suppose that the parameter $p$ in (3.2a) satisfies $p \in(0,1]$. Then, by Theorem 3.1 , for the adaptive low-gain integral controller (3.2) to achieve its objective, it is sufficient that the following two assumptions are satisfied:

(i) the semigroup generated by $A$ is exponentially stable;

(ii) $\sigma(\mathbf{G}(0)) \subset \mathbb{C}_{0}$.

It can be shown that if the well-posed system (2.2) is low-gain integral stabilizable, i.e., there exists $k^{*} \in(0, \infty]$ such that $(2.2)$ is exponentially stabilized by the integrator $\dot{u}=-k y$ for all $k \in\left(0, k^{*}\right)$, then $\sigma(A) \cap \mathbb{C}_{0}=\emptyset$ and $\sigma(\mathbf{G}(0)) \subset \overline{\mathbb{C}}_{0}$. Note that these necessary conditions for low-gain integral stabilizability are only "slightly weaker" than the sufficient conditions (i) and (ii) for adaptive low-gain integral control. Simple counterexamples (see the appendix) show that low-gain integral stabilizability does not imply either of the above conditions (i) or (ii).

(6) If, in (3.2a), $p>1$, then in general the adaptive controller fails, that is, the conclusions of Theorem 3.1 are not valid. To see this, consider the case of an exponentially stable regular single-input single-output system with $C=0$ and feedthrough $D=1$. Then $\mathbf{G}(s) \equiv \mathbf{G}(0)=D=1$. Suppose that $r=0$, so that $y(t)=u(t)$. Let $p=1+\varepsilon$, where $\varepsilon>0$. Then (3.2) becomes

$$
\begin{array}{ll}
\dot{u}=-\gamma^{-(1+\varepsilon)} u, & u(0) \in \mathbb{R}, \\
\dot{\gamma}=u^{2}, & \gamma(0)>0,
\end{array}
$$

from which it follows that $u \dot{u}=-\gamma^{-(1+\varepsilon)} \dot{\gamma}$. Integration from 0 to $t$ yields

$$
u^{2}(t)=u^{2}(0)+\frac{2}{\varepsilon}\left(\gamma^{-\varepsilon}(t)-\gamma^{-\varepsilon}(0)\right) .
$$


If $u(0)>\sqrt{2 \gamma^{-\varepsilon}(0) / \varepsilon}$, then $\lim _{t \rightarrow \infty} u(t)>0$, and so the tracking of $r=0$ is not achieved.

\section{Appendix.}

Existence of a self-adjoint positive semidefinite solution to the Lyapunov equation (3.8). It follows from the exponential stability of $\mathbf{T}$ and the admissibility of $C$ that the bilinear form $F: X \times X \rightarrow \mathbb{R}$ defined by

$$
F\left(x_{1}, x_{2}\right):=\int_{0}^{\infty}\left\langle\mathbf{T}_{t} x_{1}, \mathbf{T}_{t} x_{2}\right\rangle d t+\int_{0}^{\infty}\left\langle C_{\Lambda} \mathbf{T}_{t} x_{1}, C_{\Lambda} \mathbf{T}_{t} x_{2}\right\rangle d t
$$

is bounded. Consequently, there exists $P \in \mathcal{B}(X)$ such that $F\left(x_{1}, x_{2}\right)=\left\langle x_{1}, P x_{2}\right\rangle$ for all $x_{1}, x_{2} \in X$ (see [13, Theorem 12.8, p. 296]). It is clear that $P=P^{*} \geq 0$. Moreover,

$$
\begin{aligned}
\left\langle A x_{1}, P x_{2}\right\rangle+\left\langle A x_{2}, P x_{1}\right\rangle & =\int_{0}^{\infty} \frac{d}{d t}\left\langle\mathbf{T}_{t} x_{1}, \mathbf{T}_{t} x_{2}\right\rangle+\int_{0}^{\infty} \frac{d}{d t}\left\langle C \mathbf{T}_{t} x_{1}, C \mathbf{T}_{t} x_{2}\right\rangle \\
& =-\left\langle x_{1}, x_{2}\right\rangle-\left\langle C x_{1}, C x_{2}\right\rangle \quad \forall x_{1}, x_{2} \in \operatorname{dom}\left(A^{2}\right) .
\end{aligned}
$$

Since $\operatorname{dom}\left(A^{2}\right)$ is dense in $X_{1}, A \in \mathcal{B}\left(X_{1}, X\right)$, and $C \in \mathcal{B}\left(X_{1}, \mathbb{R}^{m}\right)$ the above identity extends to all of $X_{1}$, showing that (3.8) holds.

Counterexamples showing that low-gain integral stabilizability does not imply conditions (i) or (ii) in part (5) of Remark 3.2. Consider the finitedimensional system (with zero feedthrough) given by

$$
A=\left(\begin{array}{rr}
0 & 1 \\
0 & -1
\end{array}\right), \quad B=\left(\begin{array}{l}
0 \\
1
\end{array}\right), \quad C=(1 / 2,1) .
$$

This system is integral stabilizable (with $k^{*}=\infty$ ), but $0 \in \sigma(A)$, showing that condition (i) in part (5) of Remark 3.2 does not hold.

The finite-dimensional system given by

$$
A=\left(\begin{array}{rr}
-2 & 0 \\
0 & -2
\end{array}\right), \quad B=\left(\begin{array}{rr}
0 & -1 \\
-1 & 0
\end{array}\right), \quad C=\left(\begin{array}{ll}
1 & 0 \\
0 & 1
\end{array}\right), \quad D=\left(\begin{array}{rr}
0 & -1 \\
1 & 0
\end{array}\right)
$$

is integral stabilizable (with $k^{*}=\infty$ ). However, the transfer function is given by

$$
\mathbf{G}(s)=C(s I-A)^{-1} B+D=\left(\begin{array}{cc}
0 & -\frac{s+1}{s+2} \\
\frac{s+1}{s+2} & 0
\end{array}\right),
$$

and thus $\sigma(\mathbf{G}(0))=\{ \pm i / 2\}$, showing that condition (ii) in part (5) of Remark 3.2 does not hold.

\section{REFERENCES}

[1] P.A. Cook, Controllers with universal tracking properties, in Proceedings of the International IMA Conference on Control: Modelling, Computation, Information, Manchester, 1992.

[2] E.J. DAvison, Multivariable tuning regulators: The feedforward and robust control of a general servomechanism problem, IEEE Trans. Automat. Control, 21 (1976), pp. 35-47.

[3] R.F. Curtain and G. Weiss, Well-posedness of triples of operators in the sense of linear systems theory, in Control and Estimation of Distributed Parameter System, F. Kappel, K. Kunisch, and W. Schappacher, eds., Birkhäuser-Verlag, Basel, 1989, pp. 41-59. 
[4] A. Ilchmann and H. Logemann, High-gain adaptive stabilization of multivariable systems revisited, Systems Control Lett., 18 (1992), pp. 355-364.

[5] H. Logemann, E.P. Ryan, And S. Townley, Integral control of linear systems with actuator nonlinearities: Lower bounds for the maximal regulating gain, IEEE Trans. Automat. Control, 44 (1999), pp. 1315-1319.

[6] H. Logemann And S. TOWnley, Low-gain control of uncertain regular linear systems, SIAM J. Control Opt., 35 (1997), pp. 78-116.

[7] J. Lunze, Robust Multivariable Feedback Control, Prentice-Hall, London, 1988.

[8] D.E. Miller And E.J. Davison, An adaptive tracking problem with a control input constraint, Automatica J. IFAC, 29 (1993), pp. 877-887.

[9] D.E. Miller And E.J. DAVISOn, The self-tuning robust servomechanism problem, IEEE Trans. Automat. Control, 34 (1989), pp. 511-523.

[10] M. Morari, Robust stability of systems with integral control, IEEE Trans. Automat. Control, 30 (1985), pp. 574-577.

[11] A. PAZY, Semigroups of Linear Operators and Applications to Partial Differential Equations, Springer-Verlag, New York, 1983.

[12] W. Rudin, Real and Complex Analysis, 2nd ed., Tata McGraw-Hill, New Delhi, 1974.

[13] W. Rudin, Functional Analysis, Tata McGraw-Hill, New Delhi, 1974.

[14] D. Salamon, Realization theory in Hilbert space, Math. Systems Theory, 21 (1989), pp. 147164.

[15] D. Salamon, Infinite-dimensional linear systems with unbounded control and observation: A functional analytic approach, Trans. Amer. Math. Soc., 300 (1987), pp. 383-431.

[16] O.J. Staffans, Well-Posed Linear Systems, manuscript, 2001; also available online from http://www.abo.fi/ $\sim$ staffans/.

[17] O.J. Staffans J-energy preserving well-posed linear systems, Int. J. Appl. Math. Comput. Sci., 11 (2001), pp. 1361-1378.

[18] O.J. Staffans, Quadratic optimal control of stable well-posed linear systems, Trans. Amer. Math. Soc., 349 (1997), pp. 3679-3715.

[19] O.J. Staffans and G. Weiss, Transfer functions of regular linear systems II. The system operator and the Lax-Phillips semigroup, Trans. Amer. Math. Soc., 354 (2002), pp. 32293262 .

[20] G. WeIss, Transfer functions of regular linear systems I. Characterization of regularity, Trans. Amer. Math. Soc., 342 (1994), pp. 827-854.

[21] G. WeIss, The representation of regular linear systems on Hilbert spaces, in Control and Estimation of Distributed Parameter System, F. Kappel, K. Kunisch, and W. Schappacher, eds., Birkhäuser-Verlag, Basel, 1989, pp. 401-416. 